\section{Dental implant complications - extra-oral cutaneous fistula}

\author{
R. Mahmood, ${ }^{* 1}$ F. J. Puthussery, ${ }^{2}$ T. Flood ${ }^{2}$ and K. Shekhar ${ }^{3}$
}

IN BRIEF
- Reports a rare complication of a routinely
performed procedure.
Discusses management of an implant-
related infection.
- Highlights how vigilance is required for
extra-oral infections.

Dental implants have shown great success in recent years. However, in certain circumstances they can suffer from complications. It usually results from a combination of infection and host inflammatory responses or a lack thereof. This report documents an extra-oral cutaneous fistula associated with an osseointegrated dentoalveolar implant.

\section{CASE REPORT}

A 66-year-old lady presented complaining of pain and a lump in the left inside of her gum that had been increasing in size. She mentioned the pain was associated with the placement of dental implants approximately three months previously. On examination there appeared to be an erythematous, subcutaneous nodule on her left cheek, which was draining pus (Figs 1 and 2). Medically the patient was a type 2 diabetic and suffered from hypertension. OPG imaging demonstrated a periapical radiolucency associated with the implant in the upper left premolar region. Given the history of the presenting complaint, incision and drainage was performed, with antibiotics subsequently prescribed. On review, despite initial signs of healing, the patient began suffering from recurrent infections from the same area and a decision was made to surgically explore the area under general anaesthetic (Figs 3-5).

\section{TREATMENT}

Exploration revealed that the extra-oral sinus formed a continuous fistula to an area of fenestrated buccal bone surrounding the

'Aintree University Hospital, Liverpool, L9 7AL; ${ }^{2}$ Salisbury District Hospital, Salisbury, Wiltshire, SP2 8BJ; ${ }^{3}$ Queen Alexandra Hospital, Portsmouth, P06 3LY *Correspondence to: Dr Rizwan Mahmood Email: rizwanim@live.com

\section{Refereed Paper}

Accepted 8 May 2013

DOI: 10.1038/sj.bdj.2013.683

${ }^{\bullet}$ British Dental Journal 2013; 215: 69-70

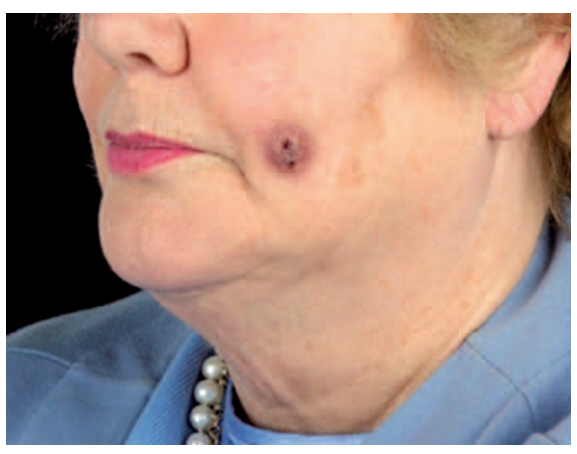

Fig. 1 Cutaneous fistula on presentation

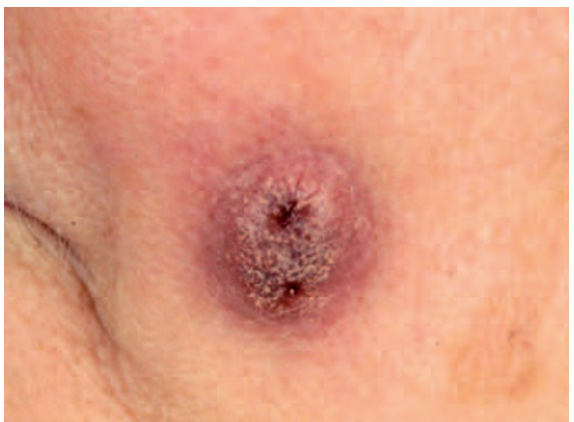

Fig. 2 Cutaneous fistula on presentation (close up)

apical region of the premolar implant, which was thought to be the source of the infection. The fistula was subsequently curetted and sent for histopathological analysis and a bioguide membrane placed over the fenestrated buccal alveolus once debridement was complete (Figs 6 and 7). The histology results revealed, as expected, a sinus tract lined with inflammatory granulation tissue.

\section{DISCUSSION}

Oral rehabilitation has been revolutionised by the advent of osseointegrated dentoalveolar implants with success rates of up

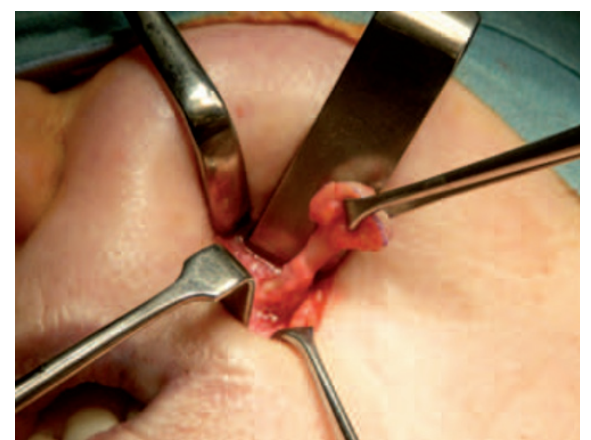

Fig. 3 Excision of cutaneous fistula

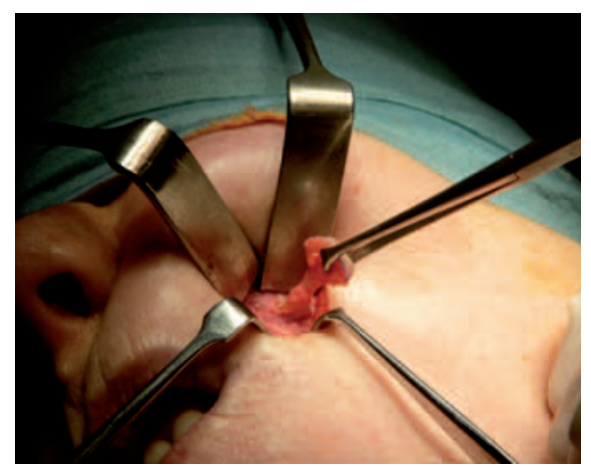

Fig. 4 Excision of cutaneous fistula

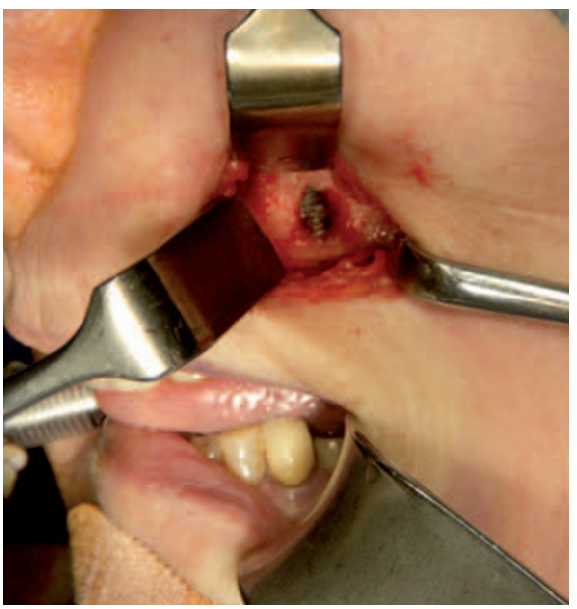

Fig. 5 Fenestrated buccal bone 
to $95 \% .^{1,2}$ Despite success rates being this high complications can arise, which can be due to a combination of several factors. These include inadequate treatment planning, oral infection, host inflammatory responses or a lack thereof. The consequences can be catastrophic as it can lead to a localised peri-implantitis, systemic infection, mobility and eventual loss of the implant. This case reports on an extra-oral cutaneous sinus tract from an osseointegrated dentoalveolar implant. To date there have been no reports on the formation of a cutaneous extra oral sinus associated with such an implant.

Radiographically there appears to be a periapical implant lesion; a cardinal sign of implant infection and possible failure. This can be caused by bone overheating, excessive tightening of the implant or overloading. ${ }^{3}$ The latter is more likely as there appears to be a radiographic fit discrepancy between the implant abutment and prosthesis. There is also some evidence to suggest that implant failure is greater in diabetic patients than the general population, ${ }^{4}$ but is not an absolute contraindication for their provision. Patients should therefore be informed of this before embarking on implant rehabilitation.

This case also highlights the significance of cutaneous sinuses, as although dental causation is rare, incorrect diagnosis can lead to ineffective and inappropriate treatment. ${ }^{5}$ This can be due to their uncommon occurrence and absence of symptoms in approximately half of individuals affected. ${ }^{6}$ It is therefore important for general practitioners to include a dental cause within a differential diagnosis for cutaneous infections of the face and neck.?

Management of the case was aimed at preservation of the implant and the authors had hoped that limited intervention would suffice. However, the presence of a persistent cutaneous infection warranted surgical intervention. According to Esposito et al. there is scope for treating implants showing signs of failure especially if the cause can be identified. ${ }^{8}$ There is also evidence to suggest that failing implants can be preserved, negating the need for removal, ${ }^{9}$ as demonstrated in this case.

Bioresorbable membranes have shown promise in bone regeneration ${ }^{10}$ and this

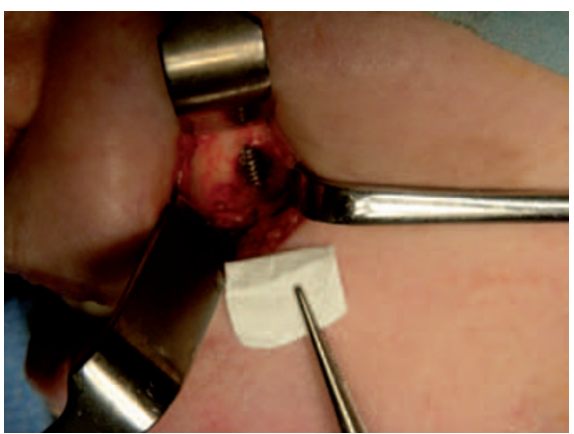

Fig. 6 Expose implant prior to "bioguide" placement

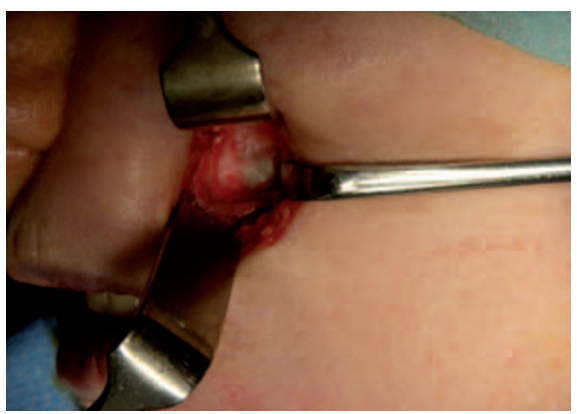

Fig. 7 Bioguide placed over exposed implant

case demonstrates the success that can be achieved with their placement to adequately facilitate bone regeneration.

Although intraoral implants have been reported to cause cutaneous infections, ${ }^{9}$ to the best of the authors' knowledge this is the only documented case of an osseointegrated dentoalveolar implant giving rise to such an infection.

\section{COMMENT}

Despite the formation of a cutaneous sinus tract, adequate debridement enabled the infected implant to be maintained. Bi-annual review indicated eradication of the cutaneous fistula with minimal scar tissue formation (Figs 8 and 9).

Implant-based oral rehabilitation is a proven form of treatment with very high success rates. However, if not appropriately planned and executed implants can result in complications and ultimately, failure. This case also demonstrates how robust they can be in the midst of a chronic infection.

Management of the infected implant should be aimed at preserving the implant if there is no acute infection, with antibiotic therapy being considered as an initial intervention. If infection persists, surgical debridement may be warranted, with evidence suggesting that the implant can still be maintained.

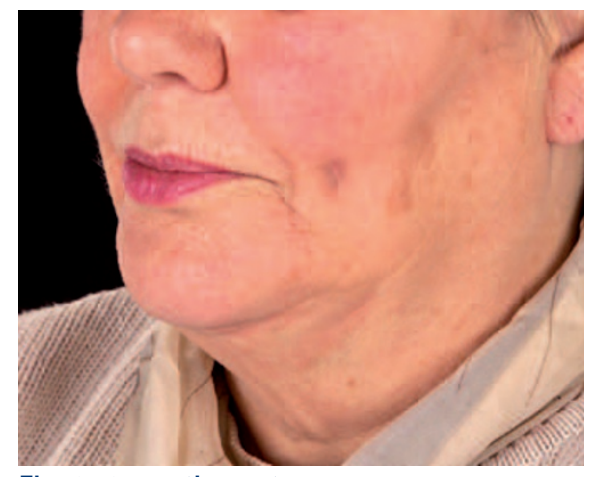

Fig. 86 months post-op

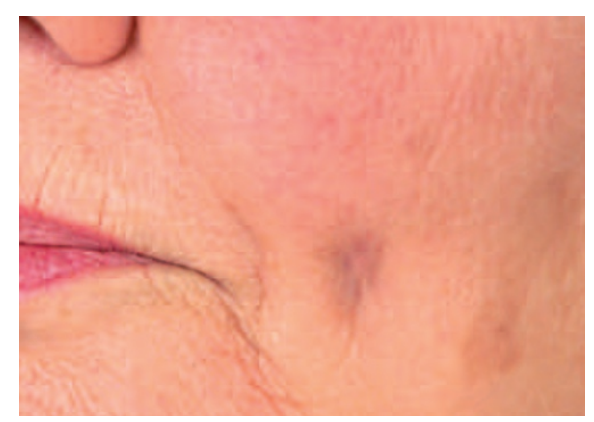

Fig. 96 months post-op (close up)

General practitioners should be vigilant of head and neck cutaneous infections and contribute an odontogenic cause within a differential diagnosis. This will ensure resources are not wasted and patients do not undergo unnecessary treatment.

1. Oliva J, Oliva $X$, Oliva J D. Five-year success rate of 831 consecutively placed Zirconia dental implants: a comparison of three different rough surfaces. Int J Oral Maxillofac Implants 2010; 25: 336-344.

2. Bahat 0 . Branemark system implants in the posterior maxilla: a clinical study of 660 implants followed for 5 to 12 years. Int J Oral Maxillofac Impants 2000; 15: 646-653.

3. Tözüm T F, Sençimen $M$, Ortakoğlu K, Ozdemir A, Aydin O C, Keleş M. Diagnosis and treatment of a large periapical implant lesion associated with adjacent natural tooth: a case report. Oral Surg Oral Med Oral Pathol Oral Radiol Endod 2006; 101: 132-138.

4. Fiorellini J P, Chen P K, Nevins M, Nevins M L A retrospective study of dental implants in diabetic patients. Int J Periodontics Restorative Dent 2000; 20: 366-373.

5. Johnson B R, Remeikis N A, Van Cura J E. Diagnosis and treatment of cutaneous facial sinus tracts of dental origin. J Am Dent Assoc 1999; 130: 832-836.

6. Cantatore J L, Klein P A, Lieblich L M. Cutaneous dental sinus tract, a common misdiagnosis: a case report and review of the literature. Cutis 2002; 70: 264-267.

7. Sheehan D J, Potter B J, Davis L S. Cutaneous draining sinus tract of odontogenic origin: unusual presentation of a challenging diagnosis. South Med J 2005; 98: 250-252.

8. Markiewicz M R, Nishiyama K, Yago K et al. Draining orocutaneous fistula associated with a failing subperiosteal implant: report of a case. J Oral Implantol 2007; 33: 347-352.

9. Mellonig J T, Griffiths G, Mathys E, Spitznagel J Jr Treatment of the failing implant: case reports. Int J Periodontics Restorative Dent 1995; 15: 384-395.

10. Tal H, Moses O, Kozlovsky A, Nemcovsky C. Bioresorbable collagen membranes for guided bone regeneration. In Tal H (ed) Bone regeneration. pp 111-138. Rijeka, Croatia: InTech, 2012. 